\title{
Impact of Alcohol Consumption on Health and Economy (A Focus on Mc Dowellization of World)
}

\author{
Dr. NTK Naik, Dr. B. Suresh Lal \\ Professor, Department of Economics, Rayalaseema University, Kurnool-AP \\ Associate Professor of Economics \& Programme Coordinator, National Service Scheme Kakatiya University, \\ Vidyaranyapuri, Warangal-506009. AP
}

\begin{abstract}
This paper focus the health impact of alcohol consumption in term of injuries, illness, disorders, including liver cirrhosis, mental illness, and several types of cancer, pancreatitis, and damage to the fetus among pregnant women and loss of economy. It also focus there is a strong correlation relation between alcohol consumer and smoker.
\end{abstract}

Submitted Date 03 .June 2013

Accepted Date: 03 .June 2013

\section{Introduction:}

Alcohol is not an ordinary commodity but a toxic substance in terms of its direct and indirect effects on a wide range of body organs and systems [1]. It is one of the most harmful risks to health. At least 61 different types of injury, illness or death, which are potentially caused by the consumption of alcohol, have been identified [2]. Adverse effects of alcohol have been demonstrated for many disorders, including liver cirrhosis, mental illness, and several types of cancer, pancreatitis, and damage to the fetus among pregnant women. Alcohol consumption is also strongly related to social consequences such as drink-driving injuries and fatalities, aggressive behaviour, family disruptions and reduced industrial productivity [3]. Approximately 2 billion people worldwide consume alcohol, around 76 million or more than one percent of whom have been estimated to be suffering from alcohol consumption disorders [4]. In countries with high prevalence of alcohol consumption, occupational productivity is seriously affected by "hangover"-related absenteeism and poor job performance [5]. Alcohol-related problems are the end result of a complex interplay between individual consumption of alcohol and the cultural, economic, physical environment, political and social contexts.

Many of our countries in Asia have not given alcohol the serious attention accorded to other addictive drugs such as narcotics and nicotine. Since the alcohol problem is not given the due urgency it warrants comprehensive information on alcohol consumption and its net effects on society are lacking in most developing and low-income countries. The reality about alcohol consumption is that the developed countries are drinking less and, in contrast, consumption in developing countries is increasing. It is falling most rapidly in the European Region and rising most rapidly in the Western Pacific Region. It is also rising in the South East Asian Region [6]. Alcohols transnational are merging and fewer of them are now supplying the bulk of the world's demands. The future's market is in the developing world, particularly Asia.

\section{Global Scenario:}

Approximately 2 billion people worldwide consume alcohol, and an estimated 76 million of them have been estimated to be suffering from alcohol consumption disorders. The World Health Assembly of May 2005 adopted resolution WHA 58.26 percent of Public health problems caused by harmful use of alcohol. According to the report on this issue by the Secretariat to the Health Assembly, "strategies and interventions in health-care settings, communities or societies at large are not equally effective in every country or society. Regional variations in average alcohol consumption and pattern of drinking mean that priorities in a country or region should be guided by available research evidence".

The Global Burden of Disease study (1997) estimated that in 1990, alcohol was responsible for 773 600 deaths, 19.3 million years of life lost and 47.7 million disability-adjusted life years lost worldwide [7]. The level of harm has been shown to be related to the pattern, including level, of drinking in a country, with overall mortality rising by 1.3 percent for every extra litre of pure alcohol consumed per capita [8]. A similar study in 2000 estimated that alcohol was responsible for 3.2percent of global deaths (estimated comparison for 1990: 1.5percent) and 4 percent of global disability-adjusted life years (DALYs) lost (estimated comparison for 1990: 3.5 percent). In 2000, alcohol consumption was estimated to have caused 1.8 million deaths in the world [9]. This indicates that the number of attributable deaths doubled while the number of years with disabilities increased marginally over the decade. Although the data also showed that some patterns of drinking lead to 
beneficial effects, i.e. preventing 300000 vascular deaths (more in women) in the established market economies of western Europe, North America and the western Pacific region, alcohol causes a total of almost 600000 vascular deaths, resulting in a net total of 268000 deaths in the world. Overall, the detrimental effects of alcohol on mortality and on disease burden in general, by far outweigh the beneficial effects. Even the beneficial effects of moderate consumption of alcohol have recently been questioned on methodological grounds [10].

\section{Asian Scenario:}

In India, Sri Lanka, Thailand, and Malaysia drinking patterns illustrate how the per capita consumption figures of a country do not necessarily give the true picture of consumption patterns of Asian countries. Parallel with the international and more expensive alcoholic beverages, there exist the local, cheap, potent brews, both legal and illicit, which are not computed into the national statistics.

In Indonesia the national consumption is only 2.7 per cent, however Balinese showed a high prevalence of 40 per cent of locally produced palm wine. In Nepal while the per capita consumption is 2.5 litres, there is a substantial amount of home production and there is no data on consumption of smuggled or home or informally produced alcohol. Alcohol is considered an integral part of most social occasions among many ethnic groups. In Sri Lanka a community survey of seven districts found 20-32 per cent current drinkers. In the rural area those who drink do so heavily. A survey in eight villages showed 71 per cent of respondents drinking daily. Use is higher among poor families. 93 per cent of respondents used locally produced alcohol, which is not reflected in the per capita consumption.

\section{Indian Scenario:}

In Western Europe there is a trend where more teenagers are turning to alcohol at a younger and younger age. This trend is also creeping into India; more teenagers are starting to drink alcoholic beverages at an earlier age. 15-20 year olds are drinking. More than 50 percent of the age group 25-35 years consumes regularly and many are drinking to excess. Of all the legal and illegal drugs, alcohol is by far the most widely used by teenagers, and according to a national survey many are regularly drinking to excess. Possibly due to liberalization of the Indian market. Several foreign companies have taken notice of India's increasing economic liberalization and have entered into joint ventures with Indian companies. Anhaeuser-Busch entered the market through a joint venture with Bombay based Shaw Wallace and Co Ltd., India's third largest brewer. Guinness entered through a joint venture with United Breweries India.

\section{Quantity Recommended:}

The U.S. National Institute on Alcohol Abuse and Alcoholism (NIAAA) has completed an extensive review of current scientific knowledge about the health effects of moderate alcohol consumption. It found that the lowest death rate from all causes occurs at the level of one to two drinks per day. That is, moderate drinkers have the greatest longevity. A 23-year prospective study of 12,000 male British physicians aged 48-78, found that overall mortality was significantly lower in the group consuming an average of 2-3 "units" (British unit = $8 \mathrm{~g}$ ) per day than in the non-alcohol-drinking group (relative risk 0.81 , confidence interval $0.76-0.87, \mathrm{P}=0.001$ ). The notable point is that the causes of death that is already known to be augmentable by alcohol accounted for only five percent of the deaths (one percent liver disease, two percent cancer of the mouth, pharynx, larynx, or oesophagus, and two percent external causes of death) and was significantly elevated only among men consuming less than two units/day.

According to Thomas A. Pearson, MD, in the year 1996 in American Heart Association scientific statement, noted, "A large number of observational studies have consistently demonstrated a U-shaped relation between alcohol consumption and total mortality. This relation appears to hold in men and women who are middle aged or older. The lowest mortality occurs in those who consume one or two drinks per day. In teetotalers or occasional drinkers, the rates are higher than in those consuming one or two drinks per day. In persons who consume three or more drinks per day, total mortality climbs rapidly with increasing numbers of drinks per day" [11].

\section{Impact on Health:}

Alcohol misuse is associated with a large range of personal, social, and health problems. Personal and societal costs include reduced vocational and educational performance, family and social disruption, and contact with the criminal justice system. Individuals are also negatively affected by other's drinking. In addition, alcohol misuse has negative economic impacts such as lost productivity, law enforcement costs, and direct health care costs. Alcohol use and abuse can contribute to a variety of medical problems, such as cardiovascular diseases, cancer, liver cirrhosis, and fetal abnormalities. Alcohol is also a major causal and contributing factor in injuries and premature deaths due to motor vehicle accidents, falls, suicides, fires, drowning, and violence. 
Alcohol abuse is one of the leading causes of death and disability worldwide. Alcohol abuse is responsible for four percent of global deaths and disability, nearly as much as tobacco and five times the burden of elicit drugs (WHO). In developing countries with low mortality, alcohol is the leading risk factor for males, causing 9.8 percent of years lost to death and disability. Alcohol abuse contributes to a wide range of social and health problems, including depression, injuries, cancer, cirrhosis, dependence, family disruption and loss of work productivity. Health and social problems from drinking often affect others besides the drinker. While men do the bulk of the drinking worldwide, women disproportionately suffer the consequences, including alcoholrelated domestic violence and reduced family budgets. Heavy alcohol use takes a particular toll on the young, and has been linked to high rates of youthful criminal behaviour, injury, and impaired ability to achieve educational qualifications. Reducing alcohol use and related problems could prevent many deaths, much disease and suffering. The risks of the alcohol consumption are given below.

\section{1).Coronary Vascular Disease.}

Moderate drinking has been found to reduce the risk of angina pectoris. In heart attack patients who are treated with alcohol, the tissues affected by low blood flow are healthier and stronger than those who receive no alcohol because of alcohol's positive effects on artery walls. Similarly, drinking alcoholic beverages in moderation may help patients recover from coronary stenting. Healing appears to be promoted by the inflammation inhibiting effects of alcohol.

\section{2).Peripheral Arterial Disease.}

Moderate alcohol consumption appears to decrease the risk of PAD in apparently healthy men. The moderate alcohol consumption was inversely associated with peripheral arterial disease in women but not in men. Residual confounding by smoking may have influenced the results. Among nonsmokers an inverse association was found between alcohol consumption and peripheral arterial disease in both men and women.

\section{3).Heart Attack and Stroke.}

Drinking in moderation has been found to help those who have suffered a heart attack survive it. To determine if moderate drinkers have fewer heart attacks because they might lead more healthful lifestyles than do abstainers or heavy drinkers, a recent reported of Harvard University, only healthy men who led healthful lifestyles. For up to 16 years the doctors monitored the health of 8,867 men who did not smoke, were of normal weight, exercised at least 30-60 minutes per day, and ate a balanced healthful diet. Among these healthy men with healthy lifestyles, those who consumed anywhere from $1 / 2$ to two alcoholic drinks of beer, wine or liquor per day had significantly decreased risk of heart attacks. Those who averaged slightly more (one to two drinks per day) had the lowest risk. Whereas abusing alcohol is associated with an increased risk of stroke. A study of over 22,000 male physicians aged 40-84 years old over an average of 12 years, concluded, "Light-to-moderate alcohol consumption reduces the overall risk of stroke and the risk of ischemic stroke in men. The benefit is apparent with as little as one drink per week. Greater consumption, up to one drink per day, does not increase the observed benefit.

\section{4).Hematologic diseases.}

Alcoholics may have anemia from several causes, they may also develop thrombocytopenia from direct toxic effect on megakaryocytes, or from hypersplenism.

\section{5).Cardiovascular Disease Coronary Heart Disease.}

Pearson reviewed the evidence supporting the effect of alcohol consumption on coronary heart disese (CHD). More than a dozen prospective studies have demonstrated a consistent, strong, dose-response relation between increasing alcohol consumption and decreasing incidence of CHD. The data are similar in men and women in a number of different geographic and ethnic groups. Consumption of one or two drinks per day is associated with a reduction in risk of approximately 30 percent to 50 percent. Studies of coronary narrowings defined by cardiac catheterization or autopsy show a reduction in atherosclerosis in persons who consume moderate amounts of alcohol. In general, the inverse association is independent of potential confounders, such as diet and cigarette smoking. Concerns that the association could be an artifact due to cessation of alcohol consumption in persons who already have CHD have largely been disproved. Another study found that when men increased their alcohol intake from very low to moderate, they significantly reduced their risk of coronary heart disease. The study monitored the health of 18,455 males for a period of seven years [12].

\section{6).Nervous System}

A meta-analysis of 35 previous studies of the effect of alcohol consumption on stroke risk found that "compared with abstainers, consumption of more than $60 \mathrm{~g}$ of alcohol per day (i.e., over four standard drinks or 
heavy drinking) was associated with an increased relative risk of total stroke, 1.64 (95 percent confidence interval. CI), ischemic stroke, 1.69 (95 percent CI) and hemorrhagic stroke, 2.18 (95 percent CI), while consumption of less than $12 \mathrm{~g} / \mathrm{d}$ was associated with a reduced relative risk of total stroke, 0.83 ( 95 percent, $\mathrm{CI}$ ) and ischemic stroke, 0.80 (95 percent CI). The meta-regression analysis revealed a significant nonlinear relationship between alcohol consumption and total and ischemic stroke and a linear relationship between alcohol consumption and hemorrhagic stroke.

\section{7).Brain development.}

Consuming large amounts of alcohol over a period of time can impair normal brain development in humans. Deficits in retrieval of verbal and nonverbal information and in visuospatial functioning were evident in youths with histories of heavy drinking during early and middle adolescence. The other way around, abstinence from chronic heavy alcohol consumption encourages new brain cell development. Nearly half of chronic alcoholics may have myopathy. Proximal muscle groups are especially affected. 25 percent of alcoholics may have peripheral neuropathy including autonomic.

\section{8).Essential tremor.}

Essential tremors can be temporarily and dramatically relieved in up to two-thirds of patients by drinking small amounts of alcohol, thus avoiding the serious side effects of the most effective and expensive medications or the dangers of surgery.

\section{9).Gallbladder disease and Gallstones.}

Consumption of alcohol is unrelated to gallbladder disease. However one study suggested that drinkers who take Vitamin $\mathrm{C}$ [ascorbic acid] might reduce their risk. "After adjustment for potential confounding variables, use of ascorbic acid supplements among drinkers was associated with a decreased prevalence of gallbladder disease and cholecystectomy. Use of ascorbic acid supplements among non-drinkers was not significantly associated with either prevalence of gallbladder disease or cholecystectomy.

The drinking alcohol reduces the risk of developing gallstones. Compared with alcohol abstainers, the relative risk of gallstone disease, controlling for age, sex, education, smoking, and body mass index, is 0.83 for occasional and regular moderate drinkers ( $<25 \mathrm{ml}$ of ethanol per day), 0.67 for intermediate drinkers $(25-50 \mathrm{ml}$ per day), and 0.58 for heavy drinkers. This inverse association was consistent across strata of age, sex, and body mass index. Frequency of drinking also appears to be a factor. "An increase in frequency of alcohol consumption also was related to decreased risk. Combining the reports of quantity and frequency of alcohol intake, a consumption pattern that reflected frequent intake (5-7 days/week) of any given amount of alcohol was associated with a decreased risk, as compared with nondrinkers. In contrast, infrequent alcohol intake (1-2 days/week) showed no significant association with risk.

\section{0).Cancer}

The National Institute on Alcohol Abuse and Alcoholism (NIAAA) reports that "Although there is no evidence that alcohol itself is a carcinogen, alcohol may act as a cocarcinogen by enhancing the carcinogenic effects of other chemicals. For example, alcohol enhances tobacco's ability to stimulate tumor formation in rats. In humans, the risk for mouth, tracheal, and esophageal cancer is 35 times greater for people who both smoke and drink than for people who neither smoke nor drink, implying a cocarcinogenic interaction between alcohol and tobacco-related carcinogens. The study determined that "3.6 percent of all cancer cases worldwide are related to alcohol drinking, resulting in 3.5 percent of all cancer deaths. The NIAAA suggests that "considerable evidence suggests a connection between heavy alcohol consumption and increased risk for cancer, with an estimated 2 to 4 percent of all cancer cases thought to be caused either directly or indirectly by alcohol".

\section{1). Urinary System.}

Studies indicate that drinking alcohol is associated with a lower risk of developing kidney stones. One study concludes, "Beer consumption was inversely associated with risk of kidney stones; each bottle of beer consumed per day was estimated to reduce risk by 40 percent. Since beer seemed to be protective against kidney stones, the physiologic effects of other substances besides ethanol, especially those of hops, should also be examined. Consumption of coffee, alcohol, and vitamin C supplements were negatively associated with stones.

\section{2).Skeletal System}

Light to moderate alcohol consumption appears to reduce the risk of developing rheumatoid arthritis, according to research. Moderate alcohol consumption is associated with higher bone mineral density in postmenopausal women. Alcohol consumption significantly decreased the likelihood. Moderate alcohol intake 
was associated with higher BMD [bone mineral density] in postmenopausal elderly women. Social drinking is associated with higher bone mineral density in men and women.

\section{3).Bacterial Infection}

Drinking strong alcoholic beverages with a meal may offer some protection against bacterial contamination. The antibacterial activity of red and white wine against enteropathogens may protect against bacterial diarrhoea in a similar way to bismuth salicylate. There is a protective effect of alcohol consumption against active infection with $\mathrm{H}$ pylori In contrast, alcohol intake (comparing those who drink > $30 \mathrm{gm}$ of alcohol per day to nondrinkers) is not associated with higher risk of duodenal ulcer.

\section{Impact on Economy:}

Alcohol advertising and promotion is rapidly expanding throughout the world and is increasingly sophisticated and carefully targeted, including to youth. It is aimed to attract, influence, and recruit new generations of potential drinkers despite industry codes of self-regulation that are widely ignored and often not enforced. Effective alcohol social policy can put into place measures that control the supply of alcohol and/or affect population-wide demand for alcohol beverages. Comprehensive policies address legal measures to control supply and demand, control access to alcohol (by age, location and time), provide public education and treatment for those who need assistance, levy taxation to affect prices and to pay for problems generated by consumption, and harm-reduction strategies to limit alcohol-related problems such as impaired driving and domestic violence. Alcohol problems are highly correlated with per capita consumption so that reductions of use can lead to decreases in alcohol problems. Because alcohol is an economic commodity, alcohol beverage sales are sensitive to prices, i.e., as prices increase, demand declines, and visa versa. Price can be influenced through taxation and effective penalties for inappropriate sales and promotion activities. Such policy measures affect even heavy drinkers, and they are particularly effective among young people. Heavy drinkers and those with alcohol-related problems or alcohol dependence cause a significant share of the problems resulting from consumption. However, in most countries, the majority of alcohol-related problems in a population are associated with harmful or hazardous drinking by non-dependent 'social' drinkers, particularly when intoxicated. This is particularly a problem of young people in many regions of the world who drink with the intent of becoming intoxicated.

In India household expenditure on alcohol varies between 3-45 per cent of their income. Alcohol misuse is one of the main killers of young men in India today. But its real impact is on the social and family dynamics that underlie its communities. Domestic violence and an exacerbation of poverty have made alcohol misuse the single most important problem for women in India. Most of the families have on roads because of alcohol drunken their husbands and now they are in debt traps. In many poor and middle class families, development taking place in one hand, their sons are becoming workers in moneylender's houses in other hand.

\section{Conclusions and Suggestions:}

This study concludes that alcohol consumption has got negative impact on human health and burden of economy.

The following are the suggestions:

- Promote the conduct, in collaboration with WHO, nongovernmental organizations, medical colleges and other academic institutions already working in this area, of well designed research and case studies to determine the magnitude, pattern and trend of alcohol consumption in the Region, and the impact for specific disease conditions and population groups.

- Develop an evidence base in order to project the magnitude of the problem on the health of the population in conjunction with other risk factors and problems, such as liver diseases, road traffic injuries and violence.

- Raise awareness of the potential for public health problems arising from alcohol consumption and the need to develop integrated strategies at national level to address the prevention and treatment of substance abuse, including alcohol.

- Support preventive interventions and awareness-raising through healthy lifestyle programmes, especially for children and youth, building on cultural and religious values to counter the glamorization of substance use, including alcohol, in the media.

- Further study is necessary to look into the matter related to alcohol consumption and its effects on health and economy, particularly in developing countries like India.

[1]. Alcohol "Bigger health threat than tobacco", The Globe Magazine 
[2]. Babor T et al, (2004); Alcohol: No Ordinary Commodity. World Health Organization and Oxford University Press

[3]. English DR et al, (1995); The quantification of drug-caused morbidity and mortality in Australia. Canberra, Commonwealth Department of Human Services and Health.

[4]. International Guide for Monitoring Alcohol Consumption and Related Harm, (2000); Geneva, World Health Organization, (Document WHO/MSD/MSB/00.4).

[5]. Global Status Report on Alcohol, (2004); Geneva, World Health Organization, 2005.

[6]. Wiese JG, Shiplak MG, Browner WS, (2000); The Alcohol Hangover. Annals of internal medicine, 132:897-902.

[7]. Murray CJL, Lopez AD, (1997); The global burden of disease: a comprehensive assessment of mortality and disability from disea ses, injuries, and risk factors in 1990\1996. Cambridge, MA, Harvard School of Public Health for the World Health Organization.

[8]. Rehm. J et al, (2003); Alcohol as a risk factor for global burden of disease. European addiction research, 9:157-64

[9]. Surveys of drinking patterns and problems in seven developing countries, (2001); Geneva, World Health Organization, (Document WHO/MSD/MSB/01.8)

[10]. Fillmore, $\mathrm{K}$ et al, (2006); Moderate alcohol use and reduced mortality risk: systematic error in prospective studies. Addiction research and theory, 14(2): 101-32.

[11]. Doll et al, (2005); Mortality in Relation to Alcohol Consumption: A Prospective Study among Male British Doctors, International Journal of Epidemiology.

[12]. Wilkie, S, (1997); Global Overview of Drinking Recommendations and Guidelines. AIM Digest, Supplement, June

[13]. The World Medical Association, (2005); Statement on Reducing the Global Impact of Alcohol on Health and Society, the WMA General Assembly, Santiago. 